\title{
DAS PATREM IM BUXHEIMER ORGELBUCH (Nr. 222)
}

\author{
Franz KÖRNDLE
}

\begin{abstract}
Since the beginning of musicological research concerning the Buxheimer Orgelbuch scholars (Schrade, Southern) have always believed the Patrem ( Nr 222) to be based on Credo IV of the Editio Vaticana, and to be incomplete. However its cantus firmus is derived from a strophic Credo created in the 14th or 15th century. The setting in the Buxheimer Orgelbuch only uses one "strophe" of this melody and therefore can be considered as complete. Liturgical documents of the time indicate that from the 14th to the 16th century existed a practice of alternatim in performing the Credo. Sometimes the sung melody ended with "et homo factus est". The organ then played the remaining part.

There exist several two-part Credos of that time as well. The beginning of the version Bayerische Staatsbibliothek München (clm 5023) is very similar to the beginning of $\mathrm{Nr} 222$ in the Buxheimer Orgelbuch. Therefore it could be that the Patrem is an intabulation of a pre-existing polyphonic setting.

Another kind of "incompleteness" of the Patrem is found in the facsimile edition of Bertha Antonia Wallner. In 1955 this facsimile was produced in which the stains found in the original manuscript had been removed. Along with one of the stains disappeared a complete chord in the Patrem.
\end{abstract}

\begin{abstract}
Abstrakt
Seit die Musikwissenschaft sich mit dem Buxheimer Orgelbuch beschäftige, glaubten die Forscher (Schrade, Southern) Patrem (Nr. 222), es basiere auf dem Credo IV der Editio Vaticana. Außerdem wurde es als unvollständig angesehen. Der Cantus firmus kommt jedoch aus einem ,,strophischen” Credo des 14. oder 15. Jahrhunderts. Davon verwendet das Patrem im Buxheimer Orgelbuch eine Strophe und kann deshalb als vollständig gelten. Liturgische Quellen aus jener Zeit lassen Rückschlüsse auf eine spezielle Alternatim-Praxis beim Credo zu. Nicht selten endete das Singen bei ,et homo factus est". Der Rest wurde von der Orgel gespielt.

Aus der damaligen Zeit sind auch mehrere zweistimmige Credo-Sätze überliefert. Die Version in clm 5023 der Bayerischen Staatsbibliothek München ähnelt in ihrem Beginn dem Patrem Nr. 222 so sehr, daß man vermuten kann, die Orgelbearbeitung sei eine Intavolierung.

Eine andere Art von Unvollständigkeit präsentiert die Faksimile-Ausgabe von Bertha Antonia Wallner. Als man 1955 ein gesäubertes Faksimile erstellte, wurden zahlreiche im Original vorhandene Flecken entfernt, einer davon befand sich im Patrem. Mit ihm verschwand ein ganzer Akkord.
\end{abstract}

Das sogenannte Buxheimer Orgelbuch (Mus. Ms. 3725 bzw. Cim 352 b der Bayerischen Staatsbibliothek, München) enthält insgesamt acht liturgische Stücke (fünf Kyrie, ein Gloria, ein Credo und ein Sanctus). Die musikwissenschaftliche Forschung hat sich damit bisher wenig befaßt, am intensivsten noch Leo Schrade in seinem 1944 erschienenen Aufsatz "The Organ in 
the Mass of the 15th Century". ${ }^{1}$ Da das Patrem hinter den anderen Sätzen an Länge deutlich zurückbleibt, wird es auch in der Erörterung nur kurz gestreift. Mit größerem Gewicht behandelte Schrade die Gloria-Bearbeitung Nr. 151 (fol. 81v-83v), wobei er den Möglichkeiten der Alternatim-Praxis von Orgel und Chor seine besondere Aufmerksamkeit widmete. Nach einem Vergleich der einstimmigen Choralmelodie mit der Orgelfassung kam er zu dem Schluß, "almost the whole Gloria ist set for Organ"”2. Diese Darstellung ${ }^{3}$, die ein Mißverhältnis von vokalem und instrumentalem Anteil suggeriert, wurde von Eileen Southern ${ }^{4}$ und Hans Rudolf Zöbeley ${ }^{5}$ übernommen. Erst Bernhold Schmid konnte überzeugend darlegen, daß mit den vom Chor vorzutragenden Tropus-Abschnitten "Spiritus et alme", der zum hier vorliegenden Marien-Gloria gehört, eine Balance der gesungenen und gespielten Teile eintritt. ${ }^{6}$ Daraus erhellt die Notwendigkeit einer genauen Kenntnis der damaligen Choralpraxis, um eine präzise Beschreibung der Aufführungsmöglichkeiten liturgischer Orgelmusik zu erlauben.

Selbstverständlich gilt dies ebenso für die bereits genannten anderen Orgelstücke zum Ordinarium Missae im Buxheimer Orgelbuch. Von diesen werde ich im folgenden den CredoSatz behandeln und dabei die verwendete Melodie sowie die Alternatimmöglichkeiten erörtern. Am Ende wird sogar die Frage zu stellen sein, ob wir es tatsächlich mit einer selbständigen Orgelbearbeitung eines choralen Credo zu tun haben, oder nicht eher mit der Intavolierung eines zweistimmigen Satzes.

Wenn das im Buxheimer Orgelbuch auf fol. 120v notierte Patrem von der musikwissenschaftlichen Forschung bisher eher am Rande behandelt worden ist ${ }^{7}$, mag das neben der bereits angedeuteten Kürze des Satzes daran liegen, daß die hier verwendete Melodie, die man dem IV. Choralcredo entnommen glaubte, nicht zur Gänze in der Komposition erscheint. Aus diesem Umstand folgerte Schrade, das Stück sei lediglich bis zum Vers "Et in unum Dominum Jesum Christum" geführt und damit unvollständig. ${ }^{8}$ Dagegen führte Eileen Southern an, das Patrem biete Mehrstimmigkeit für die Textabschnitte "Patrem omnipotentem", "Et in unum Dominum Jesum Christum", "Et ex patre natus ante omnia saecula" und "Confiteor unum baptisma".

Bevor wir der Frage nach der Vollständigkeit des Patrem nachgehen können, muß geklärt werden, ob der Tenor tatsächlich, wie Schrade und Southern übereinstimmend ${ }^{10}$ annahmen, dem Credo IV der Editio Vaticana ${ }^{11}$ entnommen ist. Obwohl von den sechs im Graduale verbliebenen

1. Leo Schrade, The Organ in the Mass of the 15th Century, MQ 28 (1944), S. 329-336 und 467-487.

2. Schrade, S. 481.

3. Erstmals und Schrade möglicherweise nicht bekannt bei Leo SöHNER, Die Geschichte der Begleitung des gregorianischen Chorals, Augsburg 1931, S. 11.

4. Eileen Southern, The Buxheim Organ Book, Institut of Medieval Music 1963, S. 119.

5. Hans Rudolf ZöBeley, Die Musik des Buxheimer Orgelbuchs. Spielvorgang, Niederschrift, Herkunft, Faktur (MVM 10), Tutzing 1964, S. $158 \mathrm{f}$.

6. Bernhold Schmid, Der Gloria-Tropus Spiritus et alme bis zur Mitte des 15. Jahrhunderts (MVM 46), Tutzing 1988, S. 110 f. Warum Southern die Möglichkeit einer tropierten Fassung verwirft, bleibt angesichts des von Schmid dargelegten Materials unklar.

7. Bei Zöbeley ist es überhaupt nicht erwähnt.

8. Schrade, S. 486.

9 Southern, S. 120.

10. Schrade, S. 486 und Southern, S. 120.

11. Graduale Triplex, Solesmes 1979, S. 776-779. 
diese Melodie unserem Tenor augenscheinlich am nächsten kommt, bleiben nämlich erhebliche Zweifel. (Notenbeispiel 1)
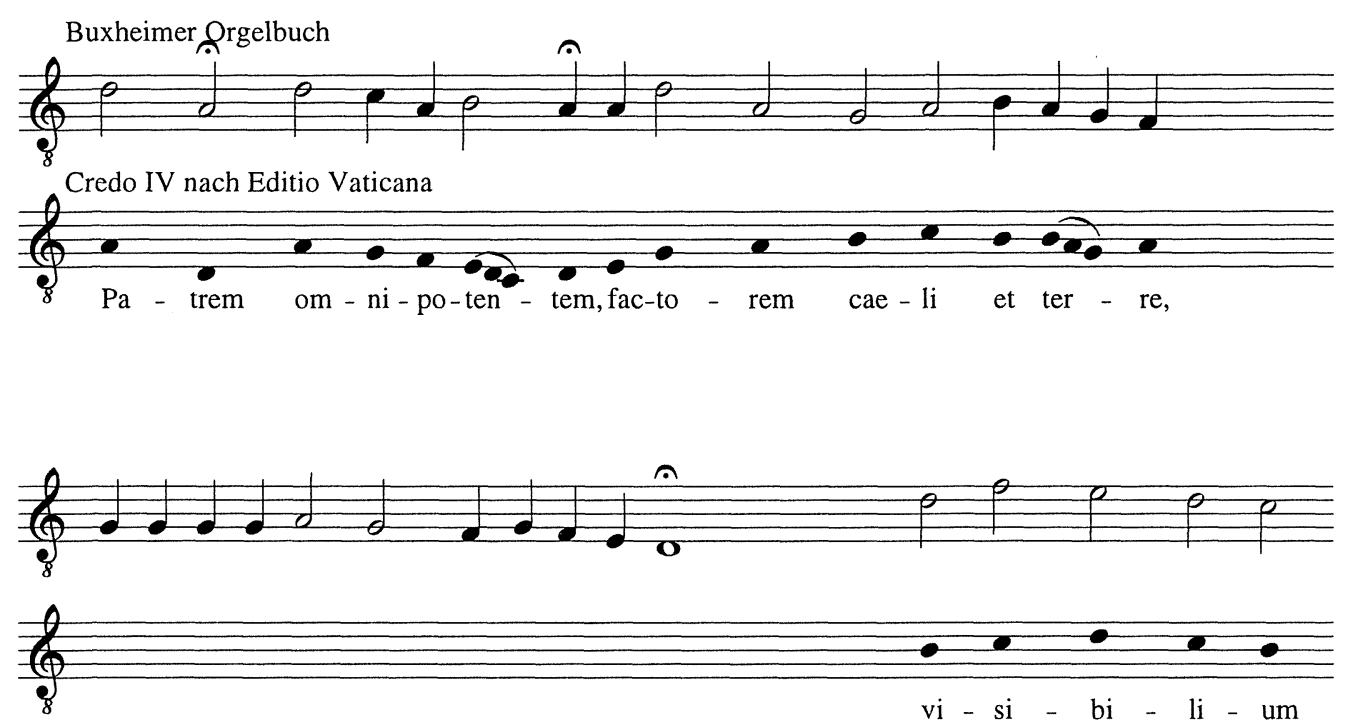

Notenbeispiel 1: Credo IV der Ed.Vat. und Tenor des Patrem

Aufgrund der unschwer sichtbaren Divergenzen beider Melodien mußte schon Schrade einräumen "the tones of the chant are hard to trace". Southern beschrieb die Abweichungen in zwei wichtigen Punkten genauer. Erstens sei der Cantus firmus eine Quarte höher als seine Vorlage notiert, ausgenommen das "Confiteor", zweitens enden die Abschnitte, wo der Choral auf der Finalis schließt, nicht eine Quarte höher, sondern eine Quinte. Dennoch seien die Konturen des Cantus firmus im wesentlichen dieselben wie im Choral. ${ }^{12}$ Sowohl Schrade als auch Southern verzichteten jedoch bei ihren Ausführungen auf eine präzise Zuordnung der Tenorglieder zu entsprechenden Abschnitten der Choralmelodie. Ein Hinweis auf das Fehlen eines Abschnittes mit mehrfachen Tonrepetitionen in Melodie IV, wie er aber im Tenor des Buxheimer Patrem erscheint, unterblieb. Ebenso ist der Analyse Southerns nicht zu entnehmen, wo die von ihr angenommene Coda am Ende des Stückes ansetzen soll. ${ }^{13}$ In der Schwierigkeit einer treffenden Zuordnung von Choral und Orgeltenor schon zu Beginn des Credo äußern sich so gravierende Abweichungen, daß dies dazu führen muß, Melodie IV als Ausgangspunkt für die Bearbeitung zu verwerfen.

1976 hat Tadeusz Miazga in seiner Studie zum einstimmigen Credo ${ }^{14}$ für die Zeit des 15. Jahrhunderts mehrere hundert Melodien nachgewiesen. In Miazgas ausführlich kommentiertem

12. SOUTHERN, S. $120 \mathrm{f}$.

13. Southern, S. 121.

14. Tadeusz Miazga, Die Melodien des einstimmigen Credo der römisch-katholischen lateinischen Kirche, Graz 1976. 
Katalog findet sich als Nr. 279 die Melodie unseres Credo IV ${ }^{15}$ und als Nr. 32 der Cantus des Buxheimer Patrem ${ }^{16}$. Beide unterscheiden sich in ihrem Tonverlauf so stark und charakteristisch voneinander, daß sie sich nicht als Varianten einer Melodie deuten lassen. Ein unwiderlegbares Argument stellt freilich die Präsenz der beiden Credo nebeneinander in mehreren Quellen dar. Sowohl Nr. 32 als auch Nr. 279 sind zuerst in oberitalienischen Handschriften des späten (?) 14. und frühen 15. Jahrhunderts anzutreffen (Monza, Bibliteca Capitolare Ms. 12 und Ms. 13, Pistoia, Archivio Capitolare, Ms. B.8), dann aber auch vielfach in osteuropäischen Cantionalien des 15. und 16. Jahrhunderts (Böhmen, Polen und Ungarn). Aus dem mittel- und süddeutschen Bereich können ebenfalls mehrere Handschriften mit umfangreicheren Credo-Faszikeln genannt werden (Erfurt, Domarchiv, Lit. 6; Nürnberg, Landeskirchliches Archiv, St. Lorenz 3; München, Bayerische Staatsbibliothek, clm 9508, clm 23001, clm 23284, clm 23286, clm 23044, clm 230045; St. Gallen, Stiftsbibliothek, Ms. 546). ${ }^{17}$

Annähernd alle Überlieferungen verwenden eine einfache rhythmische Aufzeichnungsweise, die als Cantus fractus anzusprechen ist. ${ }^{18}$ Bei dieser reduzierten Art mensuraler Notation treten als Zeichen lediglich schwarze Breven, Semibreven und caudierte Minimen auf. Das System funktioniert meist binär, gelegentlich auch ternär. Bei binärer Rhythmisierung bildet die Semibrevis den normalen Grundwert, der durch zwei Minimen ersetzt werden kann. Die Brevis erscheint nur selten.

Vor einem Vergleich des Patrem Nr. 222 mit einer der genannten Credo-Versionen von Miazga Nr. 32 muß auf einige Eigenheiten dieser Credo-Melodien eingegangen werden. Ein häufig anzutreffendes Charakteristikum ist, daß sie "unvollständig" aufgezeichnet sind. So bricht die Aufzeichnung in St. Gallen, Stiftsbibliothek 546, fol. $57 \mathrm{v}^{19}$ bei "et homo factus est" ab und schließt mit einem unmittelbar folgenden "Amen.". Bernhold Schmid hat in seinem Beitrag "Zu den Credo-Vertonungen der Benediktbeurer Handschrift 5023"20 eine genaue Beschreibung dieser Version gegeben. Für die Frage, welche Abschnitte im Buxheimer Patrem präsent sind, ist sie allerdings nur zum Teil geeignet, da hierfür eine komplette Fassung zum Vergleich unabdingbar scheint. Schmid weist jedoch auf eine neben der Verkürzung weitere wichtige Eigentümlichkeit dieser und zahlreicher anderer Credo-Melodien hin: Die Tendenz zu einer strophischen Anlage, genauer gesagt, die Aufteilung des Credo-Textes in Quasi-Strophen, die unter eine Gruppe melodischer Abschnitte unterlegt werden. ${ }^{21}$ In der Regel sind diese Credo-Melodien dennoch ausgeschrieben, so daß der strophische Bau nicht auf Anhieb zu erkennen ist. Bereits in der ältesten Credo-Melodie (I der Editio Vaticana) ist von einer ähnlichen Methode Gebrauch gemacht, nämlich den gesamten Text auf drei Tonfolge-Modelle verteilt vorzutragen.

15. MiazGA, S. 74-77.

16. Miazga, S. $45 \mathrm{f}$.

17. Nicht alle bei Miazga.

18. Otto MARXer, Zur spätmittelalterlichen Choralgeschichte St. Gallens, Freiburg/Schweiz 1908, S. 40.

19. Ediert bei Marxer, S. $169 \mathrm{f}$.

20. Musik in Bayern 33 (1986), S. 105-137, bes. S. 109-111.

21. Ebenda. 
Die Handschrift Prag, Universitätsbibliothek VII. A. 13, fol. 397v-399r gehört zu den wenigen Quellen, in denen das Credo Nr. 32 vollständig aufgezeichnet ist. ${ }^{22}$ Bei näherer Betrachtung der in Prag über vier Seiten reichenden Melodie läßt sich die hier lediglich ausgeschriebene Stropheneinteilung bereits erahnen. Erkennen kann man die Gliederung freilich erst, wenn man einen "Durchgang" bis zum melodischen Wiederaufgreifen des Beginns herausnimmt und Stück für Stück die weiteren Strophen zu unterlegen beginnt. Es zeigt sich nun, daß wir es mit zwei ineinander verschränkten Gliederungsprinzipien zu tun haben, dem melodischen Bau einerseits und der Unterteilung des Textes andererseits. Beginnen wir mit der Melodie, deren Gerüst wie folgt aussieht (Notenbeispiel 2).

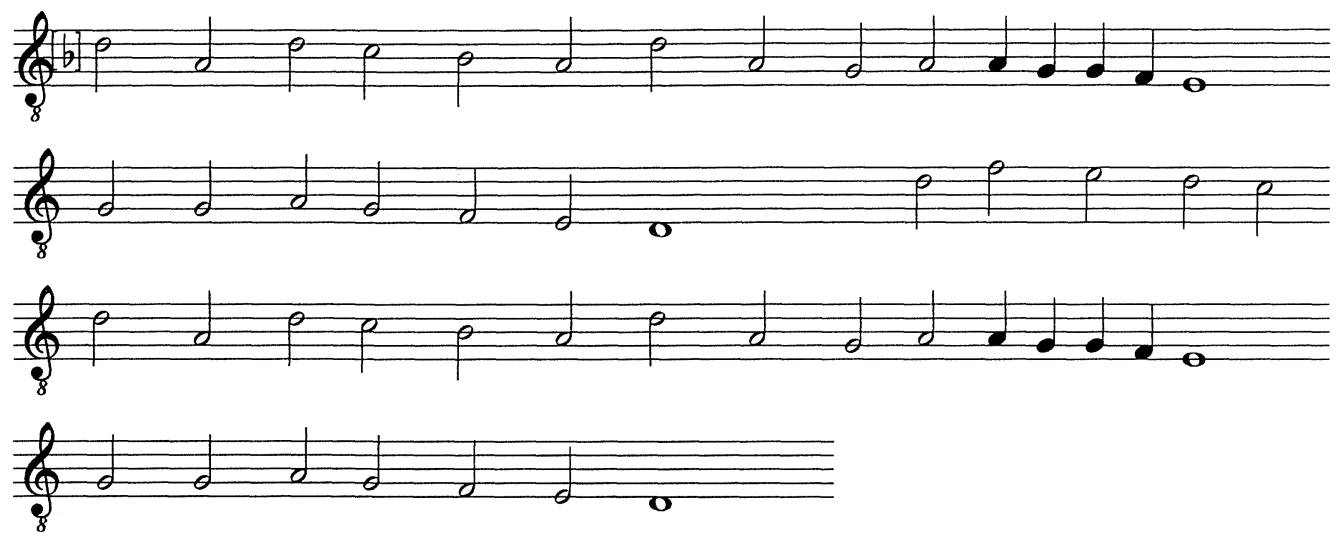

Notenbeispiel 2: Melodiegerüst des Credo Nr. 32

Es handelt sich um eine Anlage im ersten Ton, die aus zwei völlig gleichartigen Teilen besteht. Die Melodie setzt zu Beginn in der Oberoktave zur Finalis $d^{l}$ an, geht in einer ersten Phrase zur Repercussa $a$ und führt die zweite Phrase erneut vom oberen $d^{l}$ über $a$ hinunter zur Obersekund der Finalis $e$. Eine dritte, subtonal unter der Repercussa aufgehängte Phrase bringt den Abschluß auf der Finalis $d$. Der identische zweite Abschnitt ist mit dem ersten durch ein Bindeglied von fünf Semibreven verbunden, das vom oberen $d^{l} \mathrm{zu} f^{l}$ springt, um anschließend stufenweise zu $c^{l}$ abzusteigen. Nach dieser Untersekund kann die Melodie mit ihrem zweiten Teil wieder auf $d^{l}$ beginnen.

Ausgehend vom diesem Befund könnte man nun den melodischen Gesamtvorrat auf den ersten Teil plus Bindeglied beschränken. Die Textunterlegung gliedert jedoch abweichend vom

22. Václav Plocek, Catalogus codicum notis musicis instructorum qui in Bibliotheca publica rei publicae Bohemicae socialisticae in Bibliotheca universitatis Pragensis serventur, 2 Bde, Prag 1973, Bd. 1, S. 249 f. Nach Plocek stammt die Handschrift aus Böhmen vom Beginn des 16. Jahrhunderts. Die Melodie Nr. 32 erscheint in den Quellen unterschiedlich einmal mit, einmal ohne vorgezeichnetes $b$. Die von mir benützte Quelle Prag verwendet das $b$. Da das Patrem im Buxheimer Orgelbuch auf das $b$ verzichtet (darauf deutet nicht nur die unzweideutige Buchstabentabulatur für Tenor und Contratenor, sondern auch die jeweils unter einem Tenor- $h$ positionierten $e$ des Contratenor), habe ich in meiner Übertragung der Melodie Nr. 32 die Vorzeichnung eingeklammert. 
melodischen Bau dergestalt, daß keine einzige Zäsur auf den Beginn des zweiten Melodieteiles, den man bis dahin als Wiederholung auffassen konnte, fällt. Nach dem Ende des ersten Abschnitts fängt ein neuer Teil mit der Melodie des Bindegliedes an, reicht jedoch bis einschließlich zur zweiten Semibrevis der melodischen Wiederholung, die damit keine wirklich gliedernde Wiederholung mehr ist. Die nächste Textzäsur wird dann erst wieder mit dem Erreichen der Finalis gesetzt, wodurch hier die längste melodische Gruppe entsteht. Konsequenterweise entspricht der nun folgende letzte Abschnitt dieses zweiten dem letzten Abschnitt des ersten Teiles. Es wurden also aus einem melodischen Abschnitt A mit angehängter Überleitung zur Wiederholung von A zwei Teile geschaffen, deren einer als A zu benennen wäre, und deren anderer, mit der Überleitung beginnender, als B bezeichnet werden könnte. Damit ergibt sich folgendes Bild von Melodie und Textunterlegung (Notenbeispiel 3):
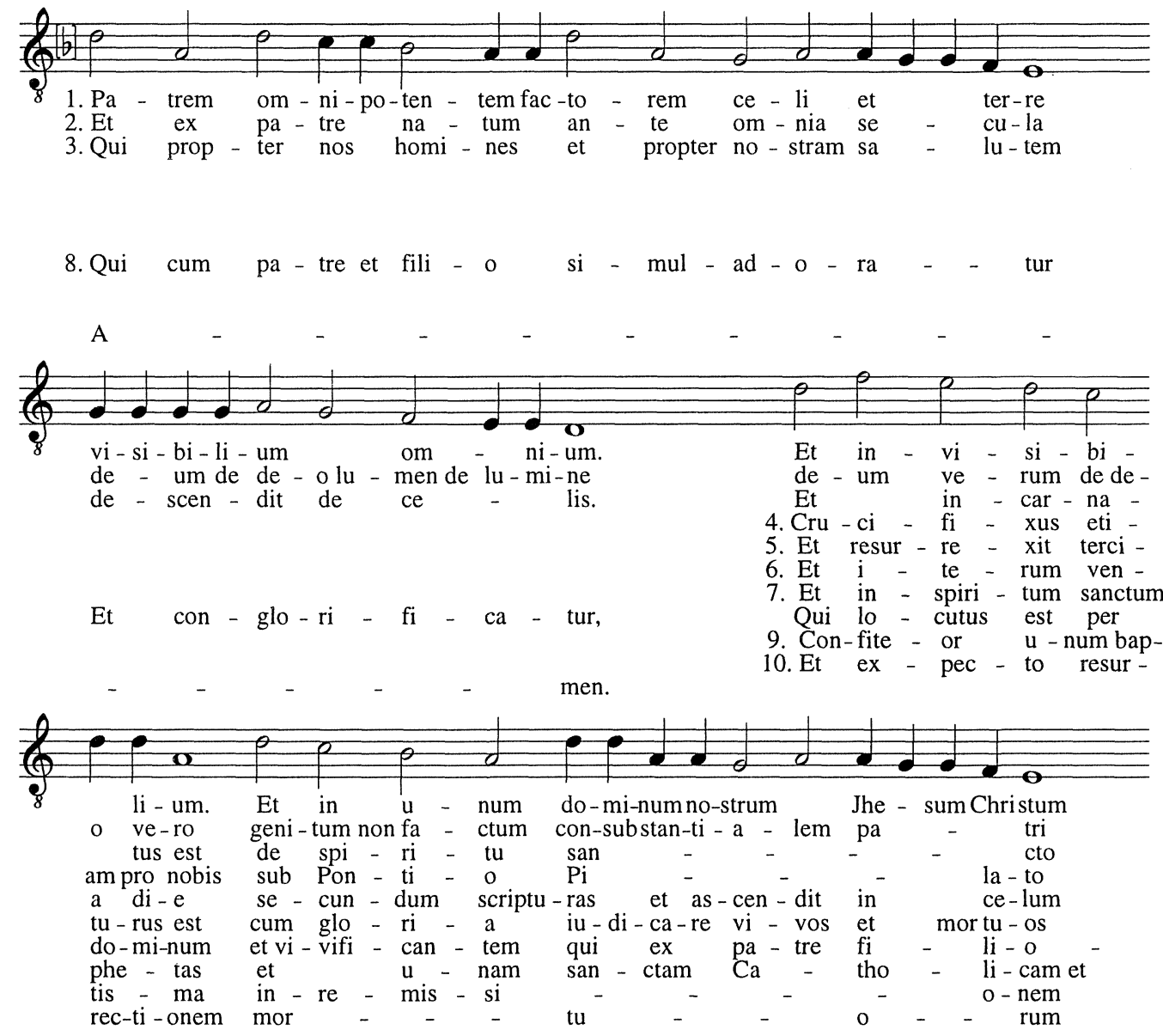


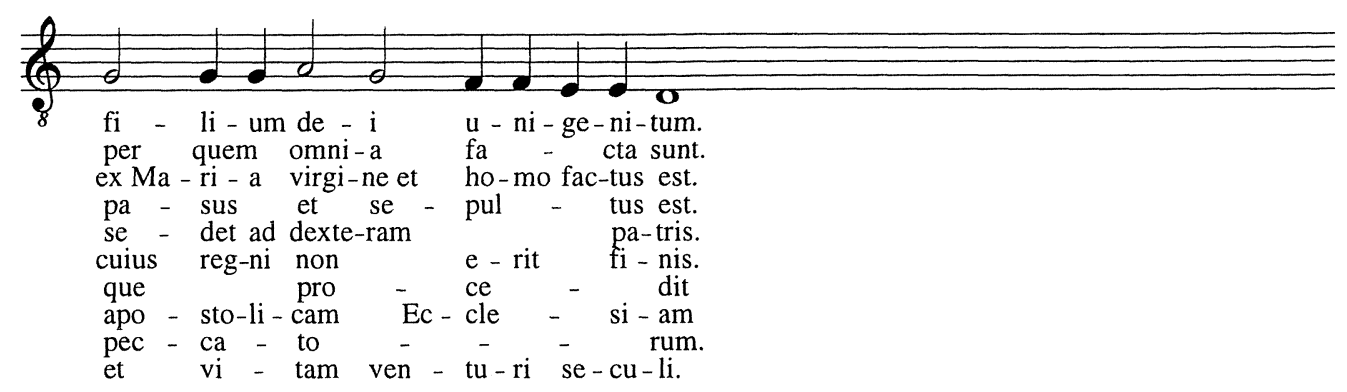

Notenbeispiel 3: Strophische Einteilung des Credo Nr. 32

Die vollständige Textunterlegung offenbart nun einen weiteren komplexen Vorgang. Lediglich die Strophen 1, 2, 3 und 8 sind Gesamtdurchläufe von A und B, dazwischen stehen "Kurzstrophen" 4, 5, 6, 7, 9 und 10, die nur Teil B beanspruchen. Den Abschluß bildet das "Amen." mit Teil A.

Ein Vergleich der Melodie Nr. 32 mit dem Tenor des Patrem aus dem Buxheimer Orgelbuch zeigt weitgehende Übereinstimmung. Allem Anschein nach basiert der Satz auf einem kompletten Durchlauf der Teile A und B. (Notenbeispiel 4)
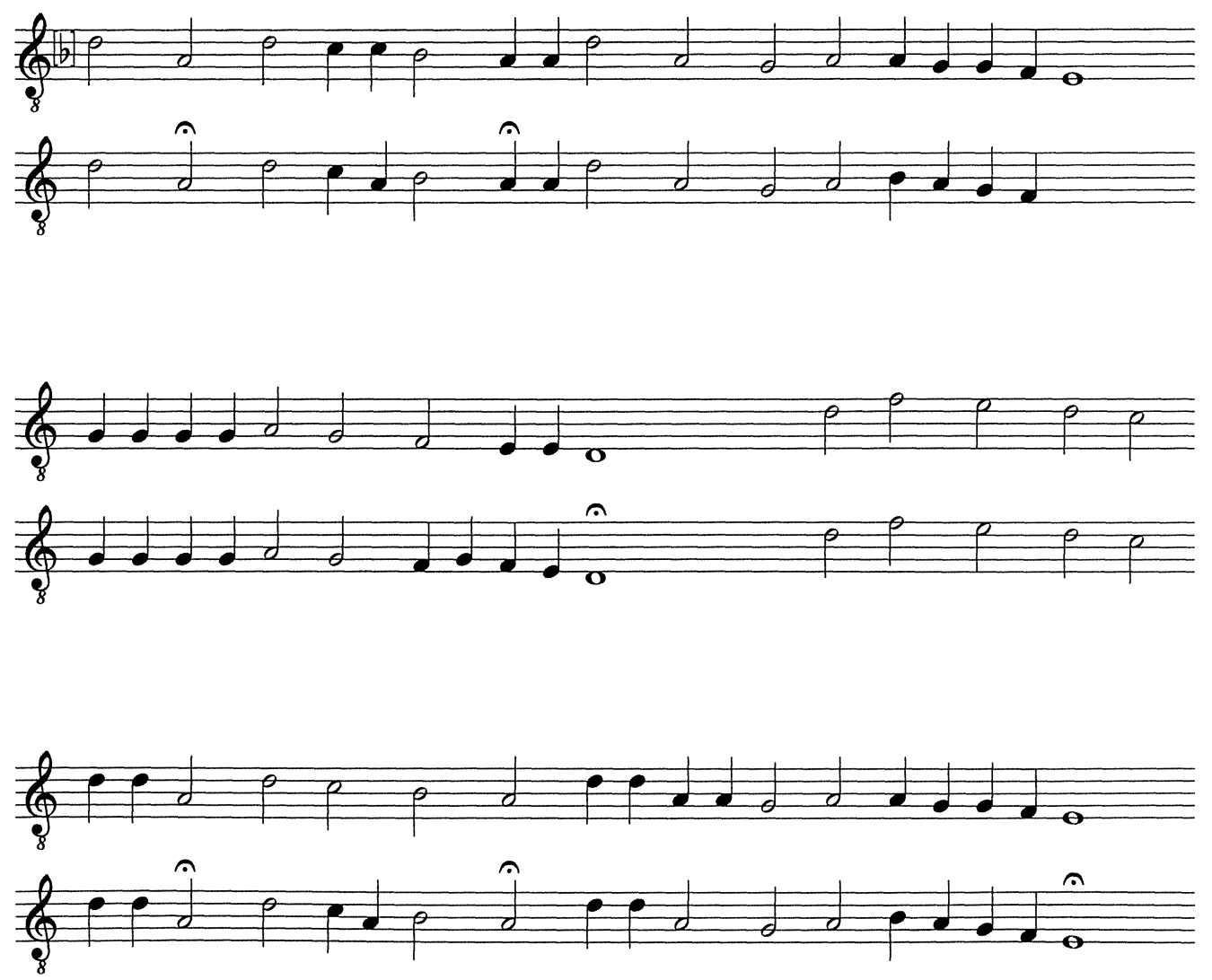


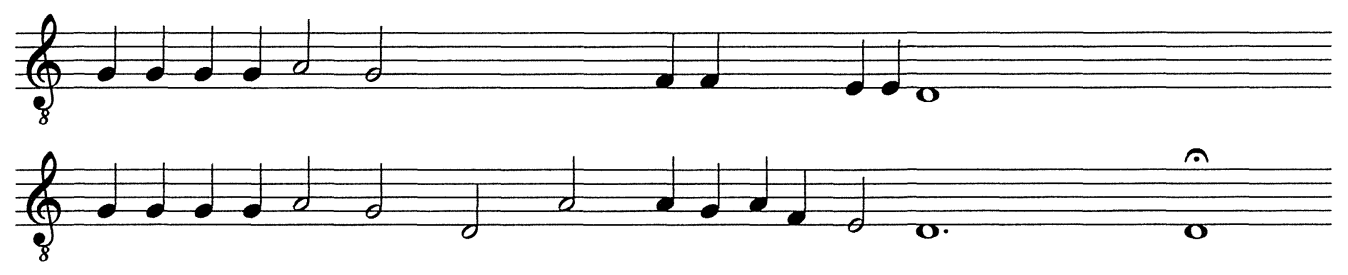

Notenbeispiel 4: Patrem aus dem Buxheimer Orgelbuch (unten) und Miazga Nr. 32 (oben)

Die eingangs aufgeworfene Frage nach der Vollständigkeit läßt sich damit wie folgt beantworten: Da das Buxheimer Patrem das komplette musikalische Material des Credo Nr. 32 in der Komposition verarbeitet, ist es als vollständig anzusehen. Dennoch bleibt offen, wie der Gesamtablauf vorzustellen wäre. Ich möchte dazu zwei Möglichkeiten skizzieren:

1. Orgelbearbeitung allein als Ersatz des gesungenen Credo, gegebenenfalls mit Wiederholung.

2 .Einstimmiger Gesang und Orgel in Alternatimpraxis wechselnd. Dabei müßte der Organist entweder

a) nur bei Komplettdurchläufen spielen oder

b) auf die "Kurzstrophen" Rücksicht nehmen oder

c) eine andere Art des Wechsels vornehmen, die sich vielleicht an den Gliederungsstrichen orientiert.

$\mathrm{Zu}$ einer genaueren Bestimmung der eventuell intendierten Möglichkeiten benötigen wir weitere Informationen. Diese lassen sich einerseits aus dem Orgelsatz selbst und andererseits aus Berichten zur liturgischen Aufführungspraxis gewinnen. Um bei der Musik selbst zu beginnen: Die Art wie der Text unter das gegebene Melodiegerüst unterlegt wird, führt bei jeder Strophe zu einer individuellen rhythmischen Lösung. Um die stark divergierenden Silbenzahlen des ja nicht genuin strophischen Credo-Textes unterbringen zu können, müssen immer wieder unterschiedlich Semibreven in zwei Minimen aufgetrennt werden. Da nun der Tenor des Buxheimer Patrem nicht allein aus Breven gebaut ist, sondern durchaus auch Folgen von Semibreven bringt, lassen sich über einen Vergleich mehrere mit dem Prager Patrem korrespondierende Passagen ermitteln. Ohne größeren Zweifel dürfte der Verfasser des Patrem-Satzes mit dem Anfang begonnen haben. Im weiteren kann der Text bis einschließlich "Et in unum Dominum nostrum Jhesum Christum" ohne Änderungen unterlegt werden. Insbesondere gibt der charakteristische Abschnitt "visibilium omnium ..." mit den vier wiederholten Minimen eine Bestätigung zu dieser Vermutung. Die nächsten Textglieder sind dem letzten verbleibenden Musikteil freilich nicht zuzuordnen. Tatsächlich findet sich in der einstimmigen Melodie nur noch ein einziges Textglied, das wiederum die vier hier nochmals auftretenden Minimen benötigt, nämlich "ex Maria virgine et homo factus est." Allerdings weicht der Buxheimer Tenor in dieser Phrase bei den folgenden Tönen stärker von der Prager Melodie-Fassung ab, so daß die weitere Textlegung ungefähr so aussehen müßte: (Notenbeispiel 5) 


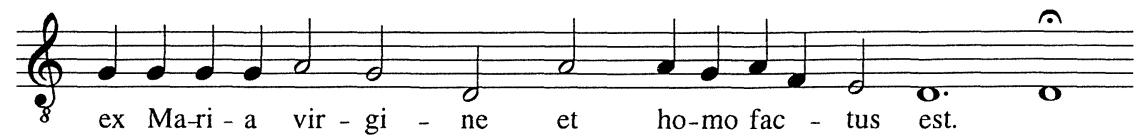

Notenbeispiel 5: letzte Zeile des Tenor aus Buxheim mit unterlegtem Text

Hier ist zurückzukommen auf die oben bereits angesprochene einstimmige Überlieferung in St. Gallen 546. Da diese ebenfalls nach "et homo factus est" abbricht, kann ein Zusammenhang der Orgelfassung mit einem solchen "Kurz-Credo" in Erwägung gezogen werden. Denkbar wäre, daß der Verfasser des Buxheimer Satzes sich von der Vorstellung hat leiten lassen, das Credo schlösse mit diesem Abschnitt. Damit wäre erklärt, warum er die angesprochene Rhythmisierung für diesen letzten Abschnitt des Tenors gewählt hat.

Einen Hinweis zur Aufführungspraxis geben Texte aus dem späten 15. und 16. Jahrhundert, die sich mit Liturgiereformen befassen. Sie beziehen sich wiederholt auf die Unart, den Vortrag des Credo an eben der genannten Stelle abbrechen zu lassen. Bereits die Synode von Schwerin vermerkt im Jahr 1492:

"Item statuimus \& mandamus, ut quilibet sacerdos nostrae dioecesis, cum gratia Dei dispositus missarum solemnia decantaverit, Gloria in excelsis, Credo, offertorium Praefationem cum Pater noster, iuxta sacrorum canonum sanctiones a principio usque ad finem decantet, nullo abstracto, diminuto, vel resecto: aut aliud responsorium, vel carmen vulgare loco praemissorum, in organis, aut choro, qui praesentes fuerint clerici, resonent. Et in Credo tractim cantetur: Ex MARIA Virgine \& Homo factus est, genibus flexis." ${ }^{23}$

Während aber hier nur sehr allgemein Kürzungen liturgischer Gesänge, darunter das Credo, untersagt werden ${ }^{24}$, formulieren die Augsburger (1548) sowie die Salzburger Reform (1562) übereinstimmend wesentlich konkreter:

"Curandum etiam est, ne sacrum diutius pertrahatur et aequo prolixius fiat et auscultantibus potius fastidium quam devotionem generet. Laudandus est dominus in organis, sed quod ipsa etiam plebs intellegit, religiosum ac pium esse. Usus vel abusus est alicubi, ut symboli pars canatur, pars organis relinquatur; praestiterit totum symbolum cani, similiter praefationem et orationem dominicam, quam partem organis relinquere." 25

23. Zitiert nach Martin Gerbert, De Cantu et Musica sacra a prima ecclesiae aetate usque ad praesens tempus, St. Blasien 1774, Bd. II, S. 198.

24. Der letzte Satz richtet sich wohl weniger gegen das Abbrechen bei "et homo factus est", sondern gegen den langsamen Vortrag dieser Textpassage. Gelegentlich geht eine Verdoppelung der Notenwerte bei dieser Stelle sogar aus dem Notenbild hervor, z.B. London, British Library, add. 10929, fol. 193v (Miazga, S. 27), wo die Notenzeichen (Punctum) nachträglich verdoppelt wurden.

25. Salzburger Reform 1562, Cap. V: De cultu divino, de liturgia et sacrificio missae, deque eius ritibus (=Augsb. Reform, Cap. XII. De Missae ceremoniis.). Zitiert nach: Alois KnöPfler, Die Kelchbewegung unter Herzog Albrecht V. München 1891, Actenstücke, S. 37. 
Wenig später forderte Carl Borromäus: "Symbolum fidei totum a choro, non alternatim ab organo, canendum decernit, perinde ac Concilium Rhemense an. 1564."26 Sogar noch im 17. und im 18. Jahrhundert finden sich ähnliche Anweisungen, die ein Fortleben der Alternatim-Praxis und der Kürzungen beim Credo belegen. ${ }^{27}$ Wie man sich das Alternatim beim Credo vorzustellen hat, zeigen mehrere andere (ebenfalls vollständige) Credo-Melodien aus St. Gallen 546, bei denen eine Aufteilung auf Organista und Chorus vorgenommen ist:

Patrem omnipotentem factorem coeli et terrae, visibilium omnium et invisibilium. Et in unum Dominum (nostrum) Jesum Christum, filium Dei unigenitum et ex patre natum ante omnia saecula. Deum de deo, lumen de lumine, deum verum de deo vero, genitum non factum consubstantialem patri per quem omnia facta sunt. Qui propter nos homines et propter nostram salutem descendit de caelis. Et incarnatus est de Spiritu sancto ex Maria virgine, et homo factus est. Crucifixus etiam pro nobis sub Pontio Pilato; passus et sepultus est. Et resurrexit tertia die secundum scripturas, et ascendit in caelum, sedet ad dexteram patris. Et iterum venturus est cum gloria, judicare vivos et mortuos, cujus regni non erit finis. Et in spiritum sanctum dominum vivificantem: Qui ex Patre Filioque procedit. Qui cum patre et filio simul adoratur et conglorificatur: qui locutus est per prophetas et unam sanctam catholicam et apostolicam ecclesiam. Confiteor unum baptisma in remissionem peccatorum. Et expecto resurrectionem mortuorum et vitam venturi saeculi. Amen.

Daß die Wechsel-Möglichkeiten sehr unterschiedlich gehandhabt werden konnten, belegt die Münchner Handschrift clm 23027, die beim Credo I auf fol. 173r nach "et incarnatus est" anmerkt "Hic cantent omnes". Gerade weil wir also aus all den genannten Quellen nicht erschließen können, wie eine Verteilung von Gesang und Orgel hinsichtlich des Patrem im Buxheimer Orgelbuch exakt ausgesehen hat, dürfen wir vermuten, daß dies vielleicht durchaus offenbleiben sollte. Und obwohl dem Verfasser für seinen letzten Tenorabschnitt der Vortrag von "ex Maria virgine..." vorgeschwebt haben dürfte, bedeutet dies keinen eindeutigen Hinweis für eine konkrete Realisierung.

Eine weitere Aufführungsvariante ist mit zwei weiteren Quellen ${ }^{28}$ für das Credo Nr. 32 zu diskutieren. In der Handschrift Mailand, Biblioteca di Brera, Ms. Ducchi di Parma, Gerli 13, fol. $1 v-5 r$ findet sich unser Credo, als tedescho bezeichnet, in einer einfachen zweistimmigen Fassung. ${ }^{29}$ (Notenbeispiel 6)

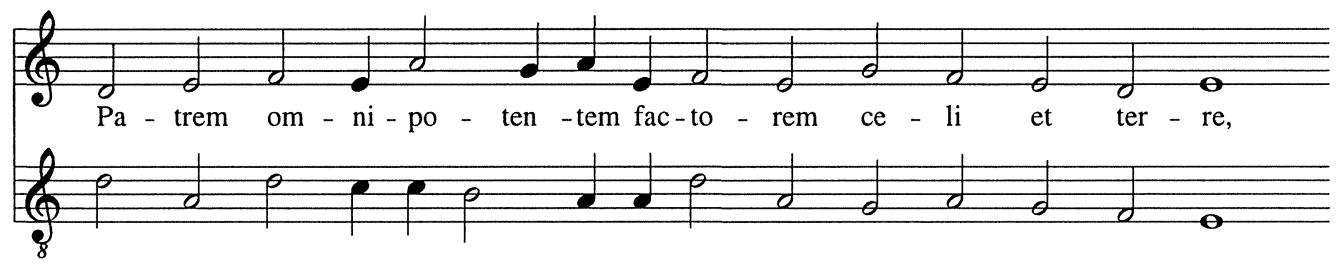

26 Gerbert, De Cantu, S. 186.

27 Gerbert, De Cantu, S. $188 \mathrm{f}$.

28 Beide nicht bei Miazga.

29 RISM B IV/4, S. 947, Nr. 1. 


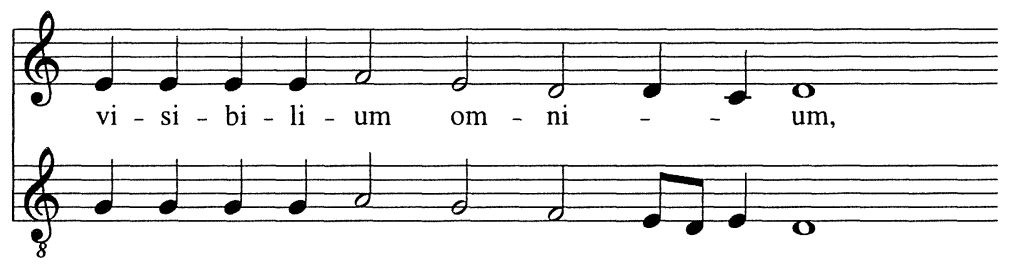

Notenbeispiel 6: Mailand, Biblioteca di Brera, Ms. Ducchi di Parma, Gerli 13, fol. 1v-5r: Credo tedescho, Beginn

Außerdem führt auch die Benediktbeurer Handschrift clm 5023 in der Bayerischen Staatsbibliothek München auf fol. 56v-57v eine zweistimmige Version des Credo Nr. 32. (Notenbeispiel 7)
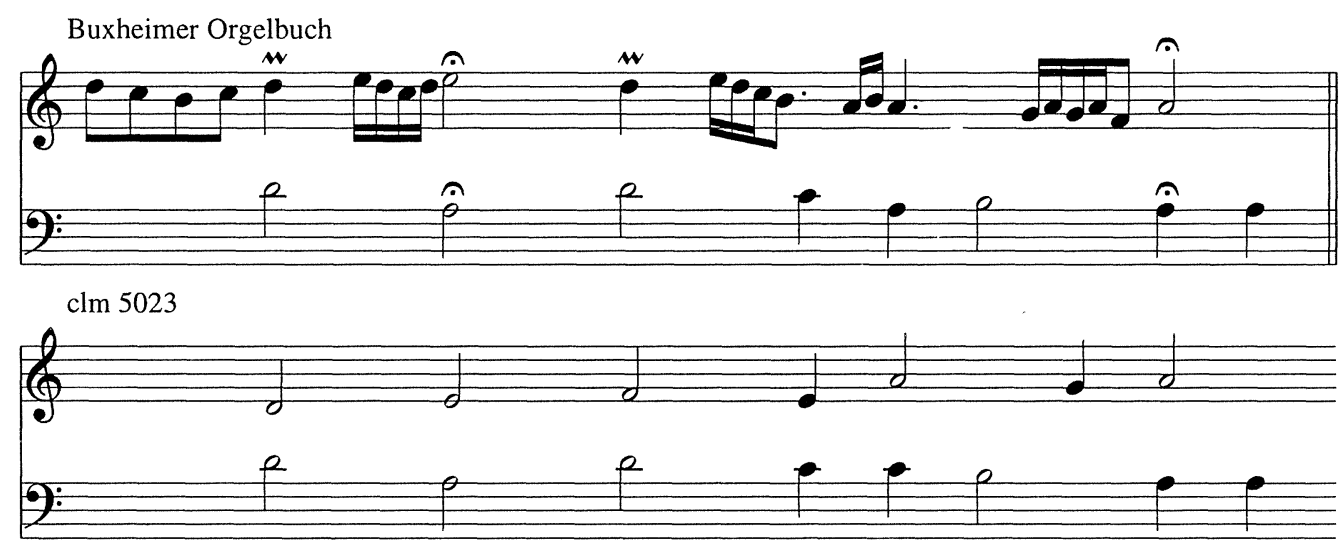

Notenbeispiel 7: Patrem aus dem Buxheimer Orgelbuch und Credo 32 aus clm 5023, Beginn

Die Art der schlichten Zusatzstimmen zu der Melodie Nr. 32 wirft die Frage auf, ob nicht ein solches zweistimmiges Credo dem Orgelsatz im Buxheimer Orgelbuch zugrunde liegen könnte. Zwar weist dieser gerade mit dem Credo aus Benediktbeuern satztechnische Ähnlichkeiten auf, doch reichen die Gemeinsamkeiten nicht hin, um eine solche zweistimmige Version als Vorlage für Buxheim anzusehen. Dennoch ist nicht auszuschließen, daß einst ein solcher Satz existiert hat, nach dem die Orgelbearbeitung geformt worden ist. Damit muß für die übrigen "liturgischen" Orgelstücke im Buxheimer Orgelbuch, die bisher als selbständige instrumentale Bearbeitungen galten, erneut der Aspekt berücksichtigt werden, es könnte sich um Intavolierungen handeln.

Abschließend ist auf die These Schrades zurückzukommen, das Buxheimer Patrem sei unvollständig. Da der Vergleich mit Melodie Nr. 32 erbracht hat, daß in der Orgelbearbeitung der 
komplette Durchlauf eines strophischen Credo ausgeführt ist, erscheint diese These in einem neuen Licht. Zwar liegt in der Tat kein Orgelsatz zum lückenlosen Credo-Text vor, sondern nur ein Ausschnitt, doch enthält dieser alle für eine durchgehende Aufführung notwendigen Elemente. Dennoch könnte man das Patrem als unvollständig ansprechen, da an der Stelle, der in der ersten Strophe mit „et terre“ endet ein Cantuston bzw, ein ganzer dreitöniger Klang zu fehlen scheint. Gegenüber der einstimmigen Version läßt der Tenor den abschließenden Ton $e$ aus. Daß wir es nicht mit einer Melodie-Variante zu tun haben, belegt der musikalische Zusammenhang: Die letzten drei Tenortöne $a-g-f$ dieses Gliedes sind in der Art des Fauxbourdon in parallelgehenden Terz-Sext-Klängen gesetzt. Der letzte dieser Klänge $f: a: d^{l}$ müßte nach den Regeln in einen Quint-Oktav-Klang $e: h: e^{l}$ als Zäsur weitergeführt werden (Notenbeispiel 8).

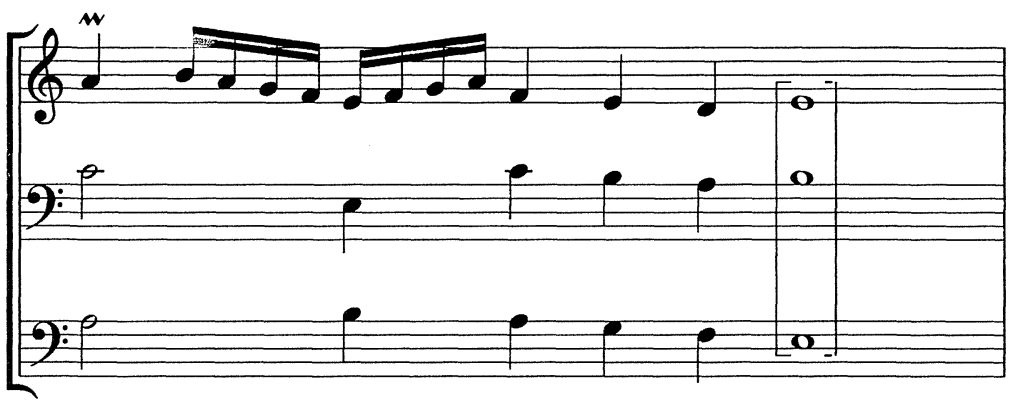

Notenbeispiel 8: “ce-li et terre" mit den Tenortönen 12-16 bzw. 17

Dieser abschließende $e: h: e^{I}$-Klang ist im Faksimile ${ }^{30}$ nicht $\mathrm{zu}$ sehen und fehlt deshalb auch in der Übertragung Wallners. ${ }^{31}$ Statt dessen beginnt in der nächsten Zeile der Handschrift ein neuer Abschnitt. Was wir hier für einen Fehler des Schreibers halten könnten, der beim Kopieren seiner Vorlage den Quint-Oktav-Kalng aus irgendeinem Grund übersehen hat, vielleicht wegen des Zeilenwechsels, ist in Wirklichkeit ein Eingriff, der beim Erstellen des Faksimiles vorgenommen wurde. Der zur Faksimilierung herengezogene Film zeigt genau an dieser Stelle einen länglichen Fleck, der den tatsächlich vorhandenen Klang $e: h: e^{l}$ überdeckte. Als man für ein „sauberes“ Faksimile den Fleck retuschierte, verschwand mit ihm auch der Klang. Mit der Wiedergewinnung dieses Klanges scheidet auch der letzte Grund aus, nach dem man das Patrem Nr. 222 im Buxheimer Orgelbuch als unvollständisg bezeichnen könte.

30. Das Buxheimer Orgelbuch: Handschrift ms. 3725 d. Bayer Staatsbibl. München, hrsg. v. B. A. Wallner (Documenta Musicologica 2;1), Kassel 1955. hier 39, S.280.

31. Das Buxheimer Orgelbuch, hrsg. van B. A. Wallner, 3 Bde. (Das Erbe deutscher Musik Bd. 37-39), Kassel 1955; 